\title{
A FRAMEWORK FOR BROKER ASSISTED VIRTUAL ENTERPRISES
}

Constantine Harbilas, Nikolaos Dragios and George Karetsos Telecommunications Laboratory, Division of Electrical and Computer Engineering National Technical University of Athens, GREECE charbil@cs.ntua.gr, ndragios@telecom.ntua.gr, karetsos@cs.ntua.gr

\begin{abstract}
A Virtual Enterprise is characterised as a temporal alliance of enterprises that come together to achieve specific common goals and tasks. The main problem for a Virtual Enterprise is the identification of the suitable enterprises to form such an alliance to achieve the desired results. This procedure includes a number of distinct difficulties that should be tackled efficiently in the antagonistic environment of the evolving global electronic economy. In this paper, we propose an open framework for the formation of Virtual Enterprises over the Internet using the notion of Information Brokers. Our study shows that an Information Broker could be quite useful in this context by acting as the constructor and administrator of such an alliance.
\end{abstract}

\section{INTRODUCTION}

A Virtual Enterprise is regarded as an information and communication infrastructure where economic organizations and individual enterprises distributed geographically and possibly not known in advance can meet each other and cooperate for a common business goal [Fielding, 1998]. VEs may be formed within a large corporation (consisting of groups located at distributed sites) or within multiple corporations as part of a business alliance or combined taskforce. Each member of a VE contributes to the alliance with its own characteristics in the creation of an entity which provides the final product or service in the best possible way in order to meet the needs of the market [Camarinha-Matos, 1997] [Cutkosky, 1996].

The main problem in the VE area is to search among many enterprises and find the appropriate partners to form an alliance for the needs of a project. Currently, there are no special tools available to facilitate the formation of VEs and usually this procedure requires the heavy involvement and supervision of humans (project managers and system operators).

In this paper, we describe how an Information Broker mediates for the automation of this procedure. Since the Broker has to represent knowledge about any activity relevant to the VE's project, its knowledge base is first referred as an essential component. The functional components of the Broker-based framework for VE creation and support are presented in the third section. A brief scenario including the steps from the formation up to the dissolution of a VE is then provided and the paper ends with future plans concerning the development of this framework. 


\section{THE NOTION OF INFORMATION BROKER}

Brokers have been proved reliable providers of information about a topic, where they are specialised, and endowed with the ability to negotiate on behalf of their clients. Travel agents are a good example of Brokerage in real life. Because of the vast amount of the available information on the Internet, the users are frequently unable to find exactly what they are looking for. Information Brokers are mentioned as a hopeful solution in this area and are mostly used in Electronic Commerce [Lambrou, 1997].

An Information Broker is the intermediate module that facilitates the exchange of information between involved stakeholders (customers and providers) using the underlying telecommunication infrastructure, by enabling providers to propagate their offers, and customers to find an offer that matches their demand.

The notion of the Broker has been extensively studied in a couple of projects we were involved. ACTS ABS [Karetsos, 1998][Tothezan, 1997] and ABROSE [Einsiedler, 1999] projects exploit most of the benefits of a Broker-based architecture. The implementation results of these projects encourage us to apply the Brokerage approach in the area of Virtual Enterprises.

First and foremost, the prospective Broker will be responsible for seeking the appropriate enterprises and taking decisions concerning the formation of the project's alliance. We currently focus on this phase but the role of the Broker can be extended in the whole life cycle of a Virtual Enterprise. It implies that the Broker can also make the rules of the alliance, control the alliance's operation, evaluate the alliance members, maintain trust between members and finally dissolve the alliance when the project is over. In general, the function of the Broker is to provide a knowledge support system for the managers and system operators involved in the alliance's project.

\section{A BROKER-BASED FRAMEWORK FOR VES}

A Broker-based framework, which must be adequate and capable to establish VEs and support them efficiently during their life cycle, is presented in this section. The consortium term has the same meaning as the Virtual Enterprise one and thus they will be used in this paper interchangeably.

\subsection{The knowledge base of the Broker}

In a business environment, such as that of Virtual Enterprises, the diversity of people, organisations or software programs is obvious. For, it is needed to provide those complex environments with the appropriate mechanisms in order that a number of trading partners to agree and define standards for their interoperation [O'Brien, 1998]. Such mechanisms should support consistent behaviour among participants, allow complex interactions such as negotiation and mediation, form a flexible, dynamic environment which does not require all participants to adopt a common infrastructure or modify an existing one [Gaines, 1995]. 
To support all the above, the Broker must be provided with all the information relative to the scope of the alliance. The Broker's knowledge base is the place where all this information is structured and stored.

It is apparent that the Broker needs to have access to sufficient and explicit information about several domains in order to be able to form a particular VE. Therefore, the Broker's knowledge base has to follow the formalities of each domain in such a way allowing the execution of statements and queries to achieve its tasks. For this purpose, a common way of naming and describing the components of each domain together with the relationships among them is essential. A common vocabulary of terms representing each specialisation area along with their relationships among them and other concepts is a prospective approach. Using ontologies is a promising methodology of such kind [Guarino, 1996][Farquhar, 1995]. However, the elaboration of ontologies for the suitable and efficient storage of knowledge items in the context of VEs is out of the scope of this paper.

At present, the approach used for the vocabulary representation is the use of one or more well-designed databases. We are referring to database(s) that is specialised for semantic-based information retrieval and results in the efficient composition of any VE by providing the appropriate environment for sharing a common interpretation format of the concepts used. Apart from that, the database also stores information about all actual enterprises that have been registered along with the characteristics and properties concerning the specialisation area of each one, what services or material provide and what kind of projects each one supports.

\subsection{Architectural framework}

In the proposed architecture an enterprise can participate in more than one consortium. This is a real situation that we often meet in real business. The participation of an enterprise in a consortium becomes active via an agent who acts on behalf of the actual enterprise. This is the Enterprise Agent (EA) who is endowed with specific roles expressing the enterprise's needs in the consortium and differs from the other roles corresponding to the same enterprise, residing on the same consortium or not. All enterprises, no matter their heterogeneous and autonomous structure, cooperate each other efficiently and contribute on the basis of the consortium. The ability of the enterprises to interact with others and at the same time keep their independence is a great challenge. At this point, we perceive the value and the usefulness of the Broker as a mediation platform for supporting the formation and operation of VEs.

Three domains are identified in the presented framework. These are the Enterprise, Broker and Knowledge domains. In the Enterprise domain, a number of individual enterprises or organisations is represented. All the required information of each enterprise is stored in the Broker domain. Any registered enterprise is then able exploit the services offered by the Broker.

In the Broker domain, the Consortiums Area is the virtual place where individual enterprises cooperate via their agents to achieve the goal for which they have formed the particular alliance. Task Administration Module generally acts as the system 
administrator for the requested tasks or commands. It operates as an initiator that receives the final list of appropriate partners and establishes the consortium.

It also includes the functional components that coordinate the enterprises' action, supports all the existing consortiums over their life cycle. During their life cycle, it coordinates the transactions and resolves any conflicts or difficulties that prevent the normal execution/operation of the consortium.

Consortium Construction Module and its components generate the list of best partners associated with various sub-goals of the initial main goal. Therefore, its responsibility is critical for the future success of the consortium. The Enterprise Profile Management receives and stores to the Knowledge domain all the data (personal data, skills, assets, technical information, etc.) that express adequately the complete profile of the enterprise in the real business life. The Consortium Construction Module evaluates this information during the partners' selection phase.

The Broker-based framework for the formation and operation of VEs is presented in detail below and the functional blocks of each module or domain are shown in Figure 1.

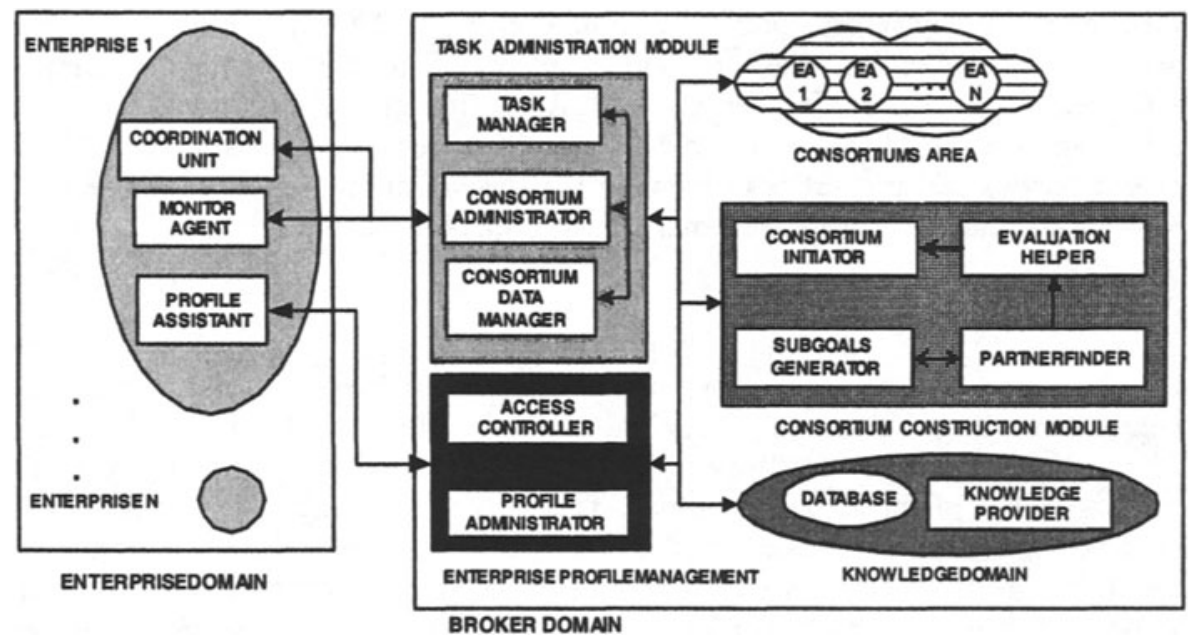

Figure 1 - Functional components of the Broker

The Enterprise domain has the appropriate graphical interfaces that help for the efficient data handling and provide a complete view of the various transactions taking place inside the consortiums. The Coordination Unit executes, updates and controls all tasks according to the goals and the evolution of the project during the consortiums' life cycle.

Communication to and from the Consortiums may be direct or indirect. In the direct way, the Coordination Unit of each enterprise interacts with its corresponding agents in the Consortiums area. The indirect way is performed via the Task Manager for monitoring purposes during the life cycle and for the formation of consortiums. Monitor Agents retrieve information from the relevant components, provide special management functions, offer on-line information of the project state, and in some 
cases, generate useful statistical data that will be helpful for the accomplishment of the management tasks. The Profile Assistant is a profile creation tool that supports identification, edition, update and deletion of enterprise profiles.

Knowledge Provider (inside the corresponding domain) administrates the database schema and organises the enterprise data. Scheduled tasks and missions of a consortium are stored and periodically updated in the databases. Special emphasis is given to the database tables containing the terms, which are extracted from the collected profiles. Their usage is very important for the Broker to discover the subgoals that are related to the user-defined goal of a new project.

Task Administrator Module comprises of the following components: Task Manager, Consortium Administrator and Consortium Data Manager. The Task Manager communicates with the Enterprise domain and helps transferring the appropriate messages and data flow into and out of the Broker. The Consortium Administrator interacts with all the existing consortiums and supervises the evolution and processes within the consortiums. This interaction provides messages and generates action points among enterprise agents during the life cycle of a VE.

The data concerning the synthesis and evolution of the consortiums are passed to the Consortium Data Manager and in turn to the Knowledge domain. Task Manager communicates with the Consortium Administrator in order to define the roles and tasks of VE members and with the Consortium Data Manager to control the data flow of the on-line data used by the monitoring agents. In the specific case that an enterprise has left a VE, the Consortium Administrator will enable the Task Manager to start a replacement phase.

Consortium Construction Module comprises the following components: Subgoals Generator, Partners Finder, Evaluation Helper and Consortium Initiator. The first component communicates with the Knowledge domain trying to decompose the main goal into more specific elements (subgoals). Partners Finder helps find all relevant partners that are specialised to each subgoal by extracting the partners data from the Knowledge Provider. The Evaluation Helper evaluates all suitable partners to select the best ones. The evaluation can be based on a constraint-based mechanism that generates a pre-defined number of the best partners for each subgoal. The project leader provides these constraints after the Broker's response about the set of proposed subgoals. After receiving a global agreement (an agreement from each proposed enterprise) the Consortium Initiator passes the entire final data list to the Consortium Administrator and establishes the consortium.

When an enterprise enters its access data, the Access Controller checks the validity to provide access. The Profile Administrator handles every operation about the profiles of the enterprises: registration, modification and un-registration. Its responsibility focuses on the extraction of the enterprise data in three parts: access, general and specific data. In the following section an explanatory scenario of the system's operation is presented.

\section{A SCENARIO OF THE BROKER'S OPERATION}

Assume GTC (Greek Technical Company) is a construction firm specialized in the buildings design and construction. Now, it is responsible for a new project: the construction of a modern building with earth-friendly technology. GTC is looking 
for the suitable collaborative firms that are specialists on various -related to the project- tasks and its experts decide on using the Broker's services to find appropriate partners to form a consortium for the completion of the particular construction.

\subsection{Registration of an enterprise}

The first step for GTC is its registration towards the Broker. GTC uses the Profile Assistant in order to publish general informational characteristics, areas of specialization and other useful data that describe its business profile. Profile Administrator stores and organizes in the Knowledge domain. New access of GTC to the Broker will be through the Access Controller. Refreshment and deletion of an existing profile follow similar to the registration steps.

\subsection{Formation of a VE}

GTC seeks for registered partners in the Broker domain capable to undertake the various tasks or parts of the project. GTC presents its goal, which is the construction of a modern building with earth-friendly technology, to the Coordination Unit on the Enterprise domain. The data flow and the procedures for the formation of such a VE are depicted in Figure 2. The goal is forwarded to Subgoal Generator via the Task Manager. Task Manager contacts Knowledge Provider to process the given goals and produce subgoals to be satisfied by other participants in the Broker Domain.

The main challenge here is a mechanism that automates the generation of the right subgoals. This gives Broker a degree of intelligence, used to exploit the knowledge representation of the Knowledge Domain to produce the set of the subgoals. Partners Finder uses the final subgoals to explore the database and find the corresponding specialists-partners. Evaluation Helper receives these results and selects the best ones per subgoal according to GTC's criteria that have been expressed as parameters in the initial goal.

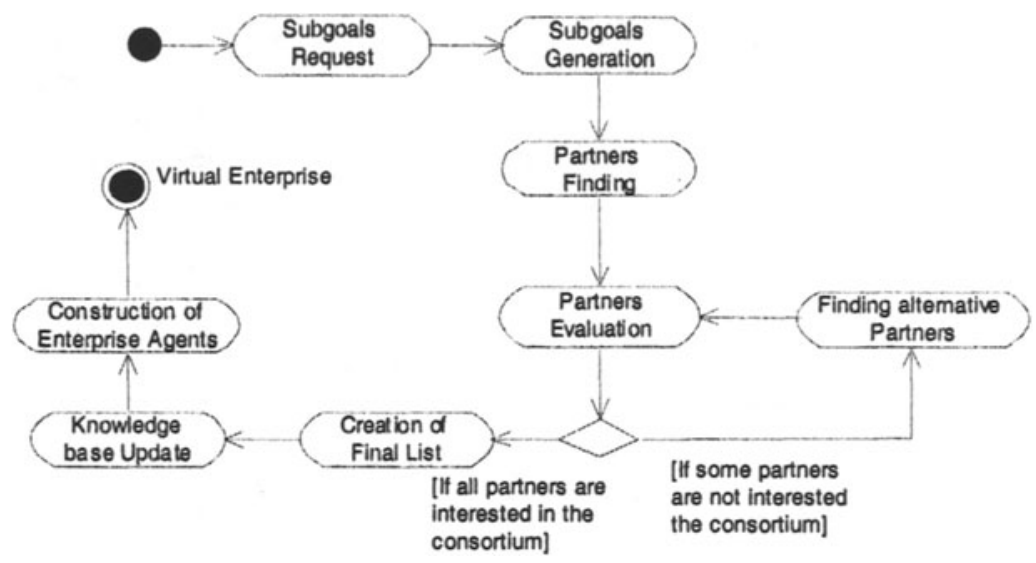

Figure 2 - Formation of a VE 
Constructor Initiator having the accepted list of partners, checks whether the selected partners desire to participate in the current project. It asks the Task Manager to broadcast a message of interest to each individual enterprise of the list. Constructor Initiator receives, in turn, all answers for every enterprise. If an enterprise is not willing to participate, it will try to receive a response from the next alternative partner.

If only positive answers are returned from partners, Consortium Initiator sends this final list of partners to the Consortium Administrator for the formation of the consortium. The consortium information is also stored in the knowledge domain by the Consortium Data Manager. Finally, the consortium is constructed and the enterprises are able to communicate directly with their own agents inside consortium.

\subsection{Life cycle support}

Consortium Administrator controls the event evolution of the project and the cooperation among the partners of all consortiums during their life cycle. The Coordination Unit of each enterprise communicates with its agents, which are always active. A Coordination Agent is recognized as the project leader. In this case, the Coordination Agent acts on behalf of GTC. The messages are transferred among all agents via the Consortium Administrator. The most important ones that have a specific or crucial role are stored in the Knowledge domain. For example, the urgent messages or the messages about the successfully finished tasks are always stored in order to produce the management plan and inform the leader's Coordination Unit and Monitor Agent.

\subsection{Replacement of a partner}

The replacement of a partner is possible during the life cycle of the consortium. Reasons for that may be: lack of materials, bankrupting, ineffectiveness or inability to achieve its mission in time. In order to replace the withdrawn partner the Broker will check the list of alternative partners. The first alternative partner is transferred to the Consortium Initiator in the Enterprise domain for its admission. If this new enterprise agrees on the terms of the cooperation of the project, the Consortium Administrator adds it to the existing VE and refreshes the data in the Knowledge domain. If things go wrong and no alternative partner is found the Broker may start the Consortium construction again. Finally, the last phase of the VE's life cycle is its termination and dissolution, which could be executed by Broker after the completion of the VE's goal.

\section{CONCLUSIONS}

In this paper, a Brokerage framework for the formation of VEs with as less as possible human intervention is presented. Other research work [Molina, $2000][$ Rabelo, 2000] proves that the Broker notion is a really promising and viable approach in Virtual Enterprises. Our proposal follows this tendency: the design and implementation of a Broker quite useful not only for the automation of the procedure of appropriate partner finding, but also for the VE's operational support. 
Further work will include the improvement of the automated formation procedure and support of VEs during their life cycle in order to facilitate project reporting and cooperation among the partners. Special emphasis will be also given to the system's knowledge base, which is going to be enhanced and best organised using of ontologies along with traditional databases.

\section{ACKNOWLEDGEMENTS}

This work was partially funded by the European IST Project SMART-EC (IST1999-10130). However, it reflects the views of the authors and not of the project as a whole.

\section{REFERENCES}

1. Camarinha-Matos L.M. and Afsarmanesh H., "Virtual Enterprises: Life cycle supporting tools and technologies", Handbook of Life Cycle Engineering: Concepts, Tools and Techniques, A. Molina, J. Sanchez, A. Kusiak (Eds.), Chapman and Hall, 1997.

2. Camarinha-Matos L.M., H.Afsarmanesh, Garita C., Lima C.: "Towards an architecture for virtual enterprises", 2nd World Congress on Intelligent Manufacturing Processes \& Systems, Budapest, June 1997.

3. Cutkosky, M. R., Tenenbaum, J. M., and Glicksman, Madefast J., "Collaborative Engineering over the Internet". Commun. ACM 39, 9 (Sep. 1996), pp. 78-87.

4. Einsiedler H.J., Barrett P., Chirichescu D., Gleizes M-P., Glize .P., Harbilas C., Léger A., Moreno J., Wilke T., "Abrose: A Co-operative Multi-Agent Based Framework for Electronic Marketplace", Infowin-Infobridge: Book about "Agent Technology", ACTS related publication, November 1999.

5. Farquhar, A., Fikes R., Pratt W. \& Rice J., "Collaborative Ontology Construction for Information Integration”, Research report 63, Knowledge System Laboratory, Stanford University, Stanford, CA, US, 1995.

6. Fielding Roy, E. James Whitehead Jr., Kenneth M. Anderson, Gregory A. Bolcer, Peyman Oriezy, Richard N. Taylor. "Support for the Virtual Enterprise: Web-based Development of Complex Information Products". Communication of the ACM, vol. 41, no. 8, pages 84-92. August 1998.

7. Gaines B.R., Norrie D., Lapsley A., "An intelligent information System Supporting the Virtual Manufacturing Enterprise", Proceedings of 1995 IEEE International Conference on Systems, Man and Cybernetics. Vancouver, Canada, October 1995.

8. Guarino Nicola, "Understanding, Building, and Using Ontologies", 10 ${ }^{\text {th }}$ Knowledge Acquisition for Knowledge-Based Systems Workshop, Banff, Alberta, Canada 1996.

9. Karetsos G.T., Lakoumentas N.K., Harbilas C.G., Katsoulas D.K and Protonotarios E.N., "An Information Brokerage Service Architecture for Electronic Commerce". NIT'98, Athens, Greece, October 1998

10. Lambrou M.A., Karetsos G.T. and Protonotarios E.N., "A Service Analysis and Service Design Framework for the Implementation of a Generic Information Brokerage Service", EMMSEC'97, Florence, November 3-5, 1997 and in the book "Advances in Information Technology: The business Challenge", pp. 226-233, IOS Press.

11. Molina A., Flores M. "Exploitation of business opportunities: the role of the virtual enterprise broker", PRO-VE'2000 $2^{\text {nd }}$ IFIP Working Conference on Infrastructures for Virtual Enterprises, Florianopolis, Brazil - 4-6 Dec 2000.

12. O'Brien Mark and Nasser Al-Biqami, "Virtual Enterprise in practice", In Proceedings of OOPSLA98 Workshop, October 1998.

13. Rabelo R.J., Camarinha-Matos L.M., Vallejos R.V., "Agent-based brokerage for virtual enterprise creation in the moulds industry", PRO-VE'2000 $2^{\text {nd }}$ IFIP Working Conference on Infrastructures for Virtual Enterprises, Florianopolis, Brazil - 4-6 Dec 2000.

14. Tothezan I., Athanassiou E., Alzon P. and Karetsos G.T., "Enterprise Modelling of Information Brokerage and Retailer Services". First international enterprise distributed object computing workshop (EDOC'97), Gold Coast, Australia, October 1997. 